\title{
Hebbian reverberations in emotional memory micro circuits
}

\author{
Luke R. Johnson ${ }^{1}$, Joseph E. LeDoux ${ }^{2}$ and Valérie Doyère ${ }^{3 *}$ \\ 1 Department of Psychiatry and Program in Neuroscience, USU, Bethesda, MD, USA \\ ${ }^{2}$ Center for Neural Science, NYU, New York, NY, USA \\ ${ }^{3}$ NAMC, CNRS-UMR8620, Université Paris-Sud, Orsay, France
}

The study of memory in most behavioral paradigms, including emotional memory paradigms, has focused on the feed forward components that underlie Hebb's first postulate, associative synaptic plasticity. Hebb's second postulate argues that activated ensembles of neurons reverberate in order to provide temporal coordination of different neural signals, and thereby facilitate coincidence detection. Recent evidence from our groups has suggested that the lateral amygdala (LA) contains recurrent microcircuits and that these may reverberate. Additionally this reverberant activity is precisely timed with latencies that would facilitate coincidence detection between cortical and sub cortical afferents to the LA. Thus, recent data at the microcircuit level in the amygdala provide some physiological evidence in support of the second Hebbian postulate.

Keywords: amygdala, fear, ensembles, network, recurrent

Edited by:

Gilles Laurent, California Institute

of Technology, USA

Reviewed by:

Shreesh P. Mysore,

Stanford University, USA

* Correspondence:

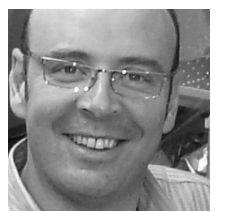

Luke R. Johnson's research program is the Micro Anatomy of Fear and Stress. He investigates the micro circuitry of the amygdala and its encoding of fear memory. He gained his PhD from Oxford University, UK. He did postdoctoral training at Yale and at NYU with Dr. Joseph LeDoux. He is currently Assistant Professor of Psychiatry and Neuroscience, and a Scientist at the Center for the Study of Traumatic Stress (CSTS) at the Uniformed Services University (USU) in Bethesda, MD, USA.

luke.johnson@usuhs.mil

\section{HEBBIAN LOGIC OF REVERBERATION AND COINCIDENCE DETECTION}

A key feature of the brain is its remarkable ability to form associations between coincident events, allowing organisms to learn from the past and thereby predict the future. When individual neurons are coincidently active they will promote a strengthening of synaptic connections. In 1949, Donald Olding Hebb (1949) proposed that if two neural signals enter the brain and simultaneously excite a post synaptic neuron, the strength of one or both of the synapses could increase such that, in the future, when only one of the signals enters the brain, that signal alone could trigger a response in the postsynaptic neuron. This proposal described a synaptic theory of associative memory (Figure 1A). The later identification of associative long-term potentiation (LTP) has provided a cellular mechanism for Hebb's first postulate. The conceptual reduction of learning to three neurons has proved tremendously informative, and extensive evidence suggests that Hebb's first postulate is a likely mechanism of learning at the level of the individual neuron (Hebb, 1949).

While most work has emphasized coincidence detection at the level of individual neurons, coincidence detection likely also occurs between neurons within micro circuits. Indeed, in addition to the idea that two coincidently active presynaptic neurons result in increased synaptic strength, Hebb also postulated that associations are distributed across ensembles of neurons. However, in the brain of a behaving organism variable latencies of coincident external signals will result because of different sensory signals via separate afferent paths, as well as separate regional processing of sensory signals. To accommodate the variable latencies and still make associations between the externally coincident events, the brain uses such tools as oscillations and neuronal ensemble activity within microcircuits, both of which can facilitate coincidence detection (Douglas et al., 1995; Johnson and LeDoux, 2004; Johnson et al., 2008; Klausberger et al., 2004; Pare et al., 2002; Pelletier and Pare, 2002; 


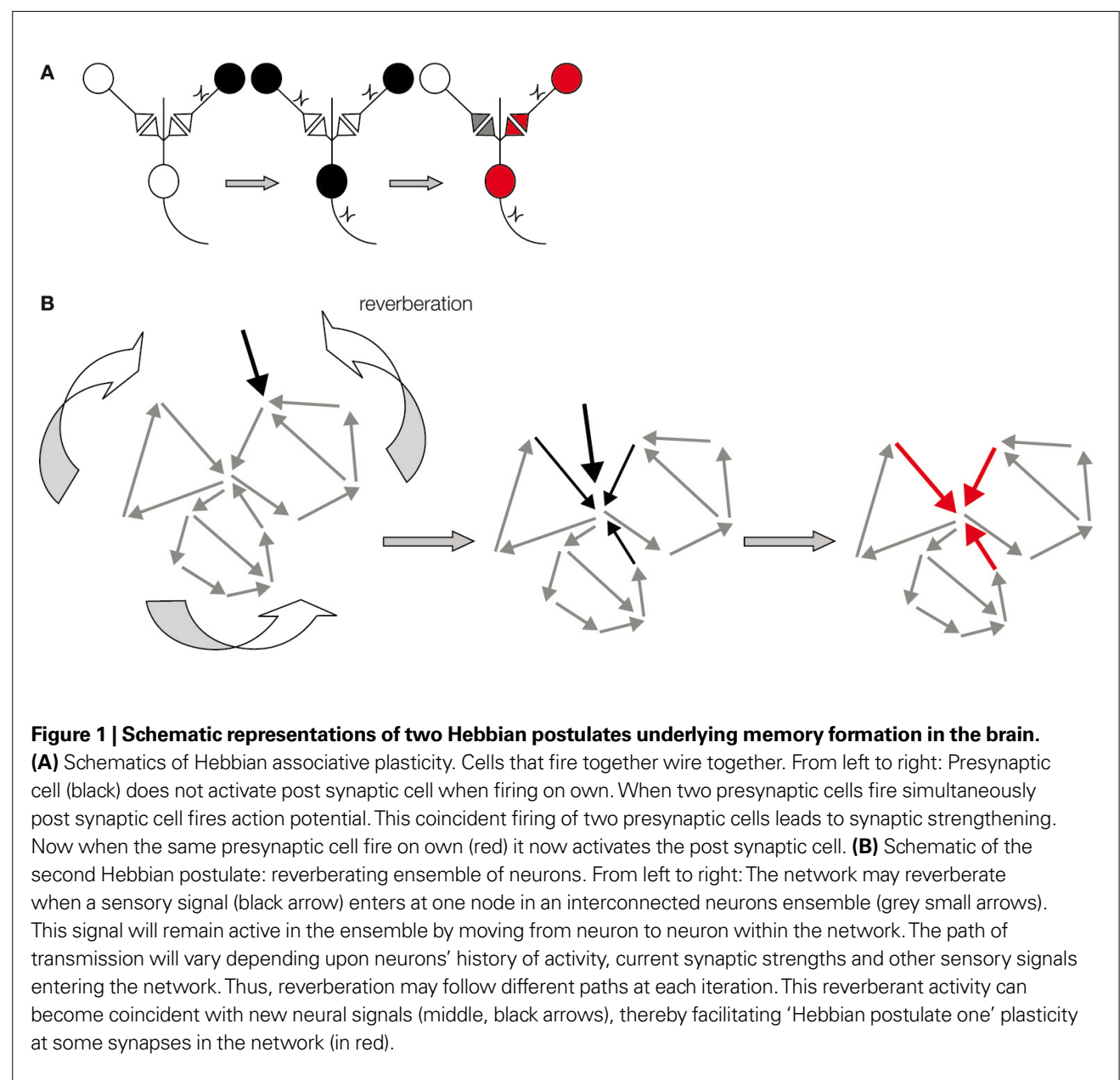

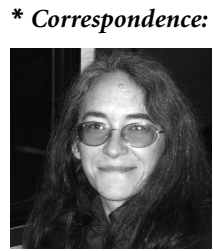

Valérie Doyère is a full-time researcher in neuroscience, with a tenure position at the CNRS since 1993. She is working in the Neurobiology of Learning, Memory and Communication lab at the University of Paris-Sud-XI, France. Her main research interest lies in synaptic plasticity (LTP, LTD) in relation with learning and memory. She records synaptic and cellular plasticity in various brain structures, including the hippocampus and the amygdala, over several days, and during learning tasks in the awake behaving rat. valerie.doyere@u-psud.fr
Seidenbecher et al., 2003; Sejnowski, 1999; Wong and Wang, 2006).

Hebb's second postulate was extrapolated from Lorente de No's original idea that reverberating networks are a fundamental organizing principle of the brain (Hebb, 1949; Sejnowski, 1999). Hebb argued that ensembles of neurons are a unit of temporary memory storage and coincidence facilitation. Thus, information can actively reverberate within the ensemble of neurons in order to maintain its activity and to coordinate additional coincidence detection of different sensory signals (Douglas et al., 1996; Hebb, 1949; Rao and Sejnowski, 2001; Sejnowski, 1999). According to Hebb, reverberant activity need not necessarily repeat in a continuous linear sequence. The ongoing neuron to neuron signaling may skip neurons and incorporate others as it cycles (Figure 1B). Thus, according to Hebbian logic, memory formation not only involves synaptic strengthening in feedforward circuits, it also involves reverberating signals in ensembles of neurons (Hebb, 1949). However, experimental evidence for functional neuronal ensembles is limited. We examined the lateral amygdala (LA) a region known for associative memory formation for evidence of reverberating neuronal ensembles (Figure 2).

\section{COINCIDENCE DETECTION IN CLASSICAL CONDITIONING OF EMOTIONAL MEMORIES}

Emotional memory and especially classical fear conditioning (also called Pavlovian fear conditioning), is one of the most described associative memory systems in neurobiology (Fanselow and LeDoux, 1999; LeDoux, 2003; Maren and Quirk, 2004; Sah et al., 2008). Conditioned fear has been observed in most animals from invertebrates to humans, and has been extensively studied in rodents (Fanselow and LeDoux, 1999; LeDoux, 2003; Maren and Quirk, 2004). Classical fear conditioning requires pairing of two sensory inputs. One input is an initially neutral stimulus, which becomes the conditioned stimulus (CS). The other input is a biologically significant event, the unconditioned stimulus (US). The pairing of 


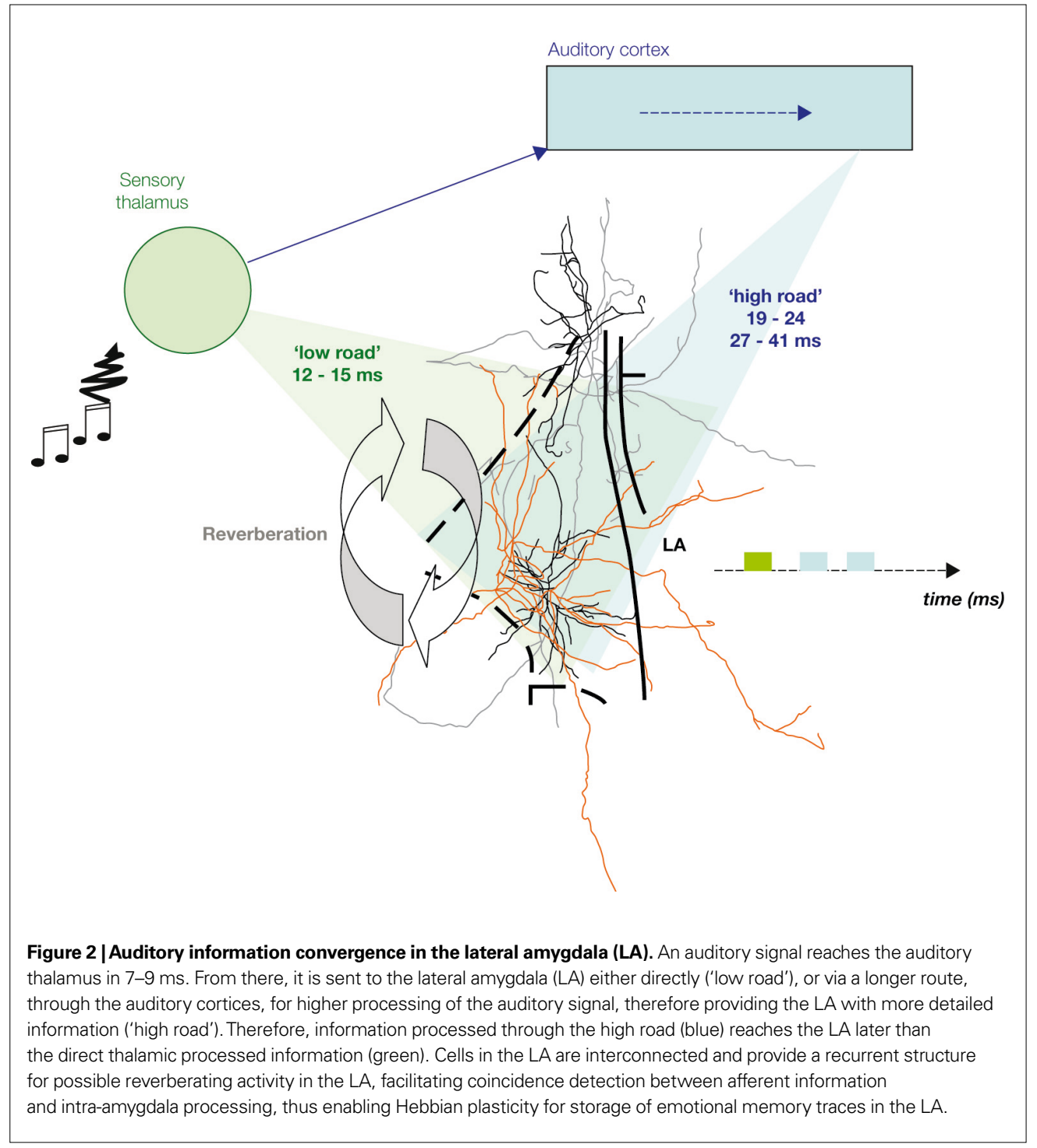

these stimuli results in a conditioned response (CR) such that the initially neutral stimulus (CS) now elicits the same behavioral response as the US (Fanselow and LeDoux, 1999; Maren and Quirk, 2004). In auditory fear conditioning a neutral auditory tone is paired with an electric shock such that presentation of the auditory tone alone elicits fear responses.

Anatomically, the two auditory pathways converge in the LA (Armony et al., 1996; Humeau et al., 2005; Romanski and LeDoux, 1992). The acoustic signal conveying the tone CS enters the LA via the auditory thalamus and the auditory association cortex (Armony et al., 1997; Romanski et al., 1993). Both of these pathways enter the LA, where they converge with somatosensory/nociceptive US signals, coming directly through the sensory thalamic complex via the spino-thalamic tract (Figure 2) (LeDoux, 2003; Maren and Quirk, 2004). The two auditory routes are generally thought to provide different aspects of the CS to the LA, with the thalamic input providing a rudimentary version of the CS ('low road', rapid) and the cortical input ('high road', slow) providing more details. While either the thalamo-LA or cortico-LA pathways is sufficient for conditioning to simple auditory stimuli, the cortical pathway appears to be required for learning about more complex stimuli (LeDoux, 2000; Pare et al., 2004).

Divergent sensory information from the same event can be processed by parallel neural paths within the brain. However, parallel neural signals may not necessarily maintain parallel latencies. The two auditory pathways to the LA both synapse onto the same LA principal neurons where 
they contact different types of dendritic spines (Humeau et al., 2005). In contrast the two auditory inputs are temporally divergent (Figure 2). The auditory input via the auditory cortex arrives some $30 \mathrm{~ms}$ later (Armony et al., 1995, 1998; LeDoux, 2000; Li et al., 1996; Quirk et al., 1997). For these two sensory signals to become rejoined in the LA one has therefore to envisage a mechanism for the brain to sustain the activity triggered by the thalamic event for sufficient time to meet the cortically processed signal. One possible mechanism that would allow for the two temporally segregated sensory inputs to converge in time as well as in space is a recurrent network within the LA. This network would allow for thalamo-LA signals to feedback to the superior parts of the LA during conditioning where they will meet incoming cortical signals (Johnson and LeDoux, 2004; Johnson et al., 2008).

Current associative plasticity models of conditioned fear rely on the concept of unidi- rectional information processing (Herry et al., 2008; LeDoux, 2003; Maren and Quirk, 2004; Pare et al., 2004; Sah et al., 2008). These models do not incorporate temporal segregation of afferent inputs (Armony et al., 1995, 1998; LeDoux, 2000; Li et al., 1996; Quirk et al., 1997). Moreover, current models do not generally incorporate the role of Hebbian reverberation or recurrent circuits in associative plasticity during conditioned fear. In order to fully understand the contribution of the LA network to conditioned fear behavior, both the structure of the LA network and its functional behavior in relation to its sensory inputs needs to be known.

\section{DETECTING NETWORK ACTIVITY IN ENSEMBLES OF LATERAL AMYGDALA NEURONS}

Recent work has shown the utility of field potentials in interpreting physiological data especially related to ensembles of neurons. These recent
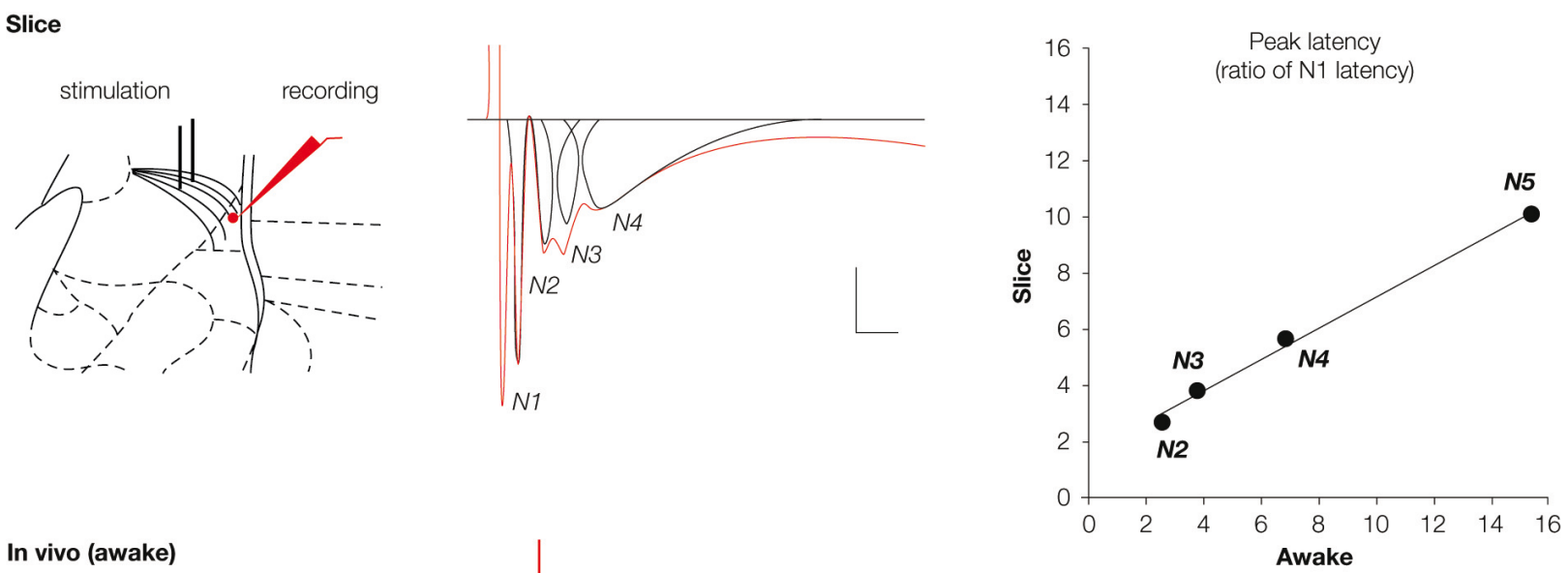

In vivo (awake)
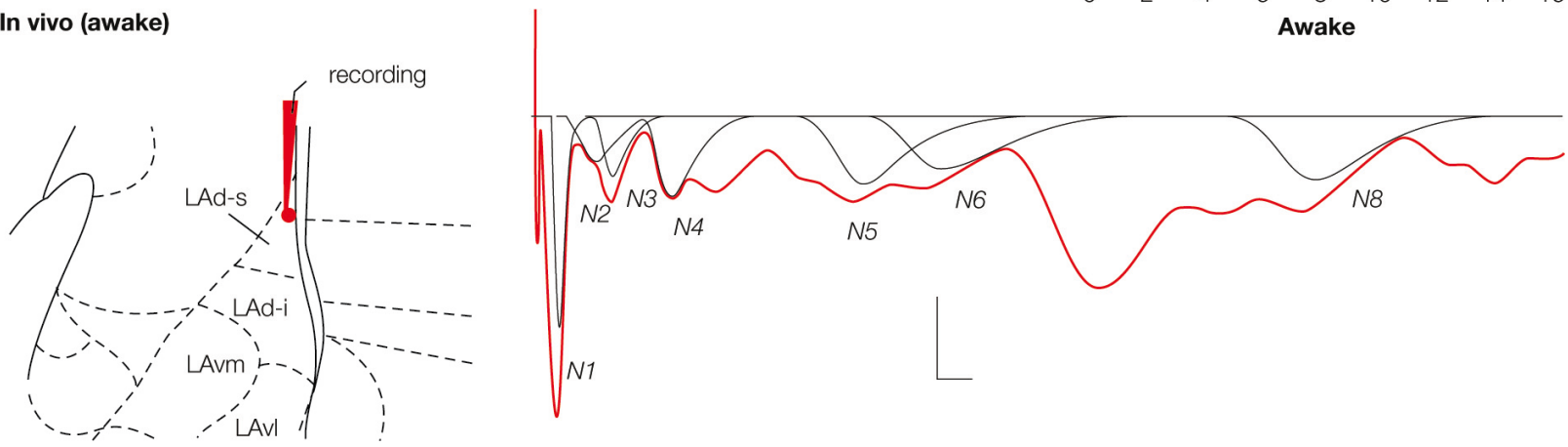

Figure 3 | Field potentials (in red) evoked in the lateral amygdala (LA) by stimulation of auditory afferents (internal capsula in vitro, auditory thalamus in vivo) showed multiple negative potentials both in vitro (slice) and in vivo (awake rat). Scale: $0.1 \mathrm{mV}, 10 \mathrm{~ms}$. These waves represent a monosynaptic response (N1) followed by polysynaptic activity. We developed a method for fitting and extracting peaks that represent reliable synchronized activity (in black). Five and nine peaks were isolated in the slice and the awake preparation, respectively. Note that not all peaks are present in a given sample (e g. here N5 is missing for the in vitro example, or N7 for the in vivo example). To enable comparison between the two preparations, latencies of each of the isolated peaks were normalized with respect to the latency of the first monosynaptic peak (N1). This transformation showed a high correlation between the two preparations ( $2=0.994 ; P<0.005)$, suggesting similar intra-network rhythmicity in both preparations, and therefore similar network behavior. 
advances have included functional measures of the discrete volume of tissue recorded in the field potential (Katzner et al., 2009; Pesaran, 2009), Additionally, increasing evidence points to the potential advantages of the field potential over single unit activity in predicting both the fMRI bold signal and importantly behavior (Goense and Logothetis, 2008; Mazzoni et al., 2008; Pesaran et al., 2002). Field potentials have been used to study plasticity in the LA but have not been used extensively to study LA network activity (Figures $\mathbf{2}$ and $\mathbf{3}$ ).

Based on the logic that reliable activity across different animals would reflect aspects of the intrinsic LA network structure we developed a method for the identification of regularity in the polysynaptic field potential peaks (Cruikshank et al., 2002; Johnson et al., 2008; Kudoh and Shibuki, 1997; Mitzdorf, 1985). We found that different network responses can be recorded at the same time from the LAd-s and LAd-i giving some measure of the localized nature of the networks recorded from. We identified both observable and hidden peaks (Figure 3) (peaks summated within larger peaks, usually indicated by changes in negative slope) from the field potential waveform using automated peak fitting. Because any single stimulation may trigger events that could represent both random and reliable responses, we used a statistical method to extract temporally invariant peaks across animals. These techniques allowed us to study both the temporal structure and plasticity in localized LA network (Figures 2-4).
B
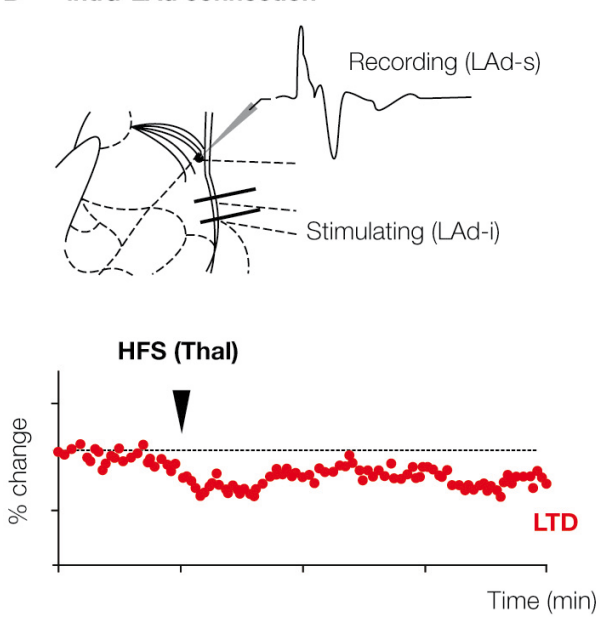

Figure 4 | Plasticity of recurrent intra-amygdala network.

(A) High-frequency stimulation (HFS) of thalamic afferents produces long-term potentiation (LTP) of the monosynaptic field potential response recorded in the superior part of the LAd, a potentiation that lasted at least $90 \mathrm{~min}$. At the same time, long-term depression (LTD) of the intra-LA network was
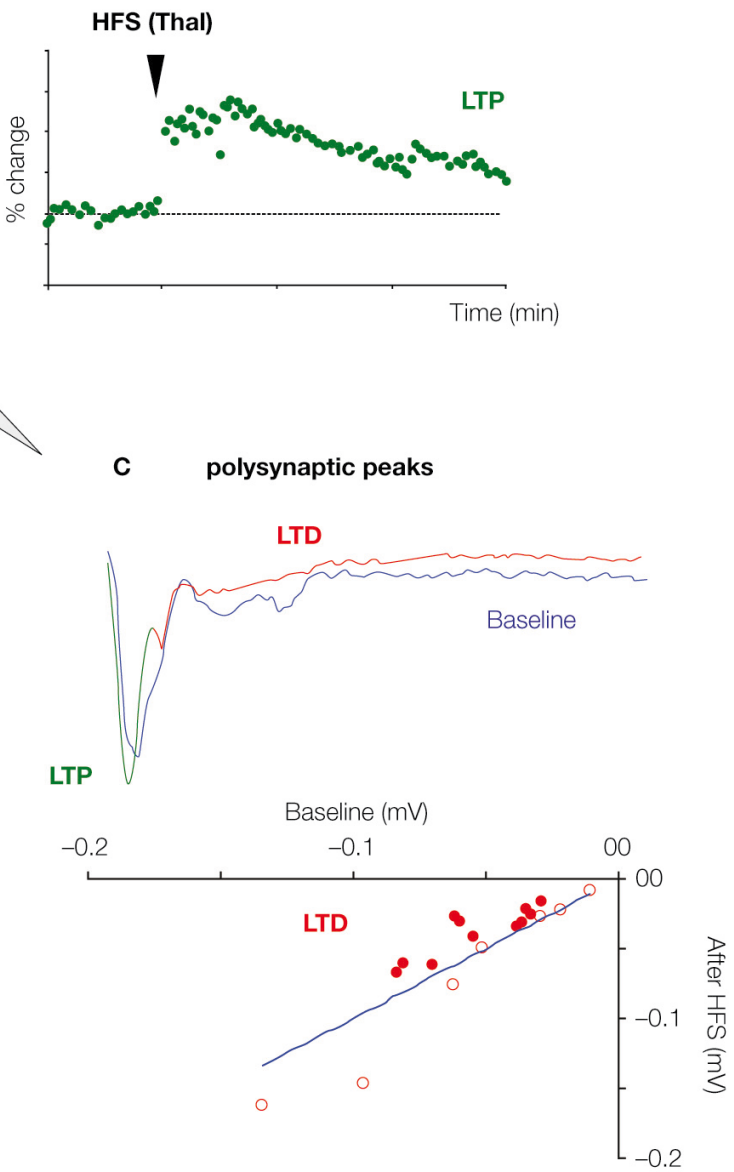

observed. This was evidenced by a depression of the monosynaptic field potential evoked in the superior part of LAd (LAd-s) in response to the stimulation of the inferior part of the LAd (LAd-i) (B); This was also observed as a general decrease in the amplitude of polysynaptic peaks (filled red dots), representing LTD in the network activity (C) 


\section{RECURRENT AND REVERBERATING NETWORKS WITHIN THE LATERAL AMYGDALA}

The LAd most likely contains a network of interconnected neurons. Thalamic afferents enter the LAd from a dorsomedial direction and then apparently course in a ventral direction through the LA (Doron and Ledoux, 1999, 2000; LeDoux et al., 1990, 1991). This is the direction of neural activity and information flow considered in current models (Herry et al., 2008; LeDoux, 2003; Maren and Quirk, 2004; Pare et al., 2004; Sah et al., 2008). In contrast, in the network we have identified, neural activity appears to propagate in all directions throughout the LAd. Importantly this propagation also includes an apparent recurrent feed back from the inferior to superior parts of LAd (Figure 2). This network can reverberate when triggered by stimulation of thalamic to LA afferents. Reverberating activity can be observed to last for up to $40 \mathrm{~ms}$ in vitro and in the awake rat apparent polysynaptic activity can be observed to continue beyond $240 \mathrm{~ms}$.

The reverberating LAd network appears to behave in a structured manner. Using a new method for extracting signatures of network activity from the extracellular field potential, we measured time-locked mono and polysynaptic activities as peaks in the field potential traces (Johnson et al., 2008). We named these peaks sequentially from the monosynaptic negative peak N1 to polysynaptic peaks N2 through N5 (N1 to N9 in vivo) (Figure 3). We localized the origin of each of these peaks to either the superior or inferior LAd (LAd-s, LAd-i). Upon stimulation of thalamic afferents in vitro, monosynaptic activity is detected first in the LAd-s and then $3 \mathrm{~ms}$ later in the LAd-i. After the initial monosynaptic activation of the LAd, polysynaptic activity is seen throughout. In the LAd-s the initial polysynaptic activity $\mathrm{N} 2$ is mediated by 'feedback' from the LAd-i. Following N3 and N4 peaks appear to be local to the LAd-s because trans-section of the LAd-s and LAd-i did not affect these peaks. Finally, polysynaptic peak $\mathrm{N} 5$, like $\mathrm{N} 2$, is also derived from the LAd-i because it is also lost after the mid LA trans-section. As a confirmation, correlation of latencies from polysynaptic peaks obtained from the LAd-s with and without LAd-i trans-section reveals a best fit regression when peaks $\mathrm{N} 2$ and N5 are considered absent.

The same pattern of polysynaptic excitatory events appears in vivo in the awake rat. Upon stimulation of the medial geniculate nucleus of the thalamus, both mono and polysynaptic peaks are observed in the extracellular field potential. The polysynaptic peaks are also negative peaks which follow the negative monosynaptic field excitatory post synaptic potential (fEPSP). The latency of the peaks is longer than that observed in the in vitro preparation. However, while the latencies are not identical the inter-peak relationship follows a matching pattern in the vivo and in vitro preparations. This is evident by the very close correlation between in vitro and in vivo patterns of peak latencies (Figure 3). Given the matching pattern of polysynaptic peaks in vivo and in vitro, at least for the first 93 and $39 \mathrm{~ms}$ respectively, one interpretation is that they may reflect the same or similar intrinsic network structures. The logic of this idea is that in vitro brain $400 \mu \mathrm{m}$ thick slice will contain large sections of the same network that exists in vivo. Moreover, stimulating the same thalamo amygdala afferent pathways will trigger the same intrinsic network activity. Since field potentials are thought to measure network activities within a $250 \mu \mathrm{m}$ radius (Katzner et al., 2009; Pesaran, 2009), it is plausible that the network identified in $400 \mu \mathrm{m}$ thick slices in vitro is the same as the one identified in vivo.

The temporal organization of the recurrent LAd network is consistent with known corticoamygdala latencies. An auditory tone, as used in conditioned fear, triggers in the LA, first a fast monosynaptic response via thalamic afferents, followed by a later monosynaptic response via cortical afferents. Therefore these auditory thalamic and cortical responses in the LA are separated by time. Our in vitro and in vivo data suggest that between the thalamic and cortical monosynaptic events, precisely timed recurrent activity reverberates within the LAd. Both the recurrent N2 and the LAd-s local N3 are active within LAd-s at the same time as the activity from the monosynaptic cortical input (Armony et al., 1995; Li et al., 1996). This provides a mechanism for convergence across time of the thalamic and cortical sensory information. This converging information will include elements of the same original sound that is processed directly to LA via the thalamus, as well as via the cortex. In addition, it is also possible that other sensory information, for example tone and light, as well as aspects of the tone and shock could also show temporal converge via the reverberating LA network. Thus, the reverberating and recurrent networks of the LAd provide a mechanism to facilitate coincident interaction between cortical and subcortical information.

\section{PLASTICITY WITHIN HEBBIAN LATERAL AMYGDALA NETWORKS}

The second Hebbian postulate of reverberating ensembles incorporates network plasticity. This 
plasticity can be in two forms. First, the temporal coordination of sensory signals in reverberating ensembles facilitates convergence dependent upregulation of synaptic strength (seen as LTP). In contrast, reverberating ensembles may also facilitate the intra-network weakening of synaptic strength (seen as LTD). Both LTP and LTD are supposed to play a critical role in the storage of memory, by increasing contrast between strengthened and weakened synapses, Synaptic weakening likely plays a critical role in networks by preventing saturation and refining the memory trace. In addition, as some synapses within the network are strengthened, the increased activity in the network could ultimately lead to run away excitation. Therefore some mechanism of inbuilt synaptic weakening is believed necessary for network homeostasis.

Such homeostatic plasticity is identifiable in the synapses of the LAd network. LTP in the thalamic to LA pathway leads to LTD in the LAd-i to LAd-s pathway (Figures 4A, B). If the polysynaptic network is the same recurrent network, changes in the polysynaptic peaks would also be predicted. Induction of LTP in the thalamic to LA pathway leads to LTD in the polysynaptic peaks. Peaks N2 through N5 reduced in amplitude following monosynaptic LTP (Figure 4C). This plasticity within the reverberating LA network provides further evidence the network may contain key features of Hebbian reverberating ensembles.

Another feature of recurrent networks which has been studied more recently is that they appear to utilize NMDA receptor mediated mechanisms to promote propagation of activity (Tegner et al., 2002; Wang, 2001). Interestingly, several key behaviors have been shown to be dependent upon NMDA receptors, and therefore may be based on key aspects of recurrent reverberating network activity. For example, NMDA receptors at recurrent CA3 to CA3 synapses have been shown to underlie feedback and are essential for the CA3 mediated pattern completion of spatial cues in a water maze task (Nakazawa et al., 2002). In addition, blockade of NMDA receptors in the LA disrupts second order fear conditioning (Gewirtz and Davis, 1997), a fear learning based on the association between two different CSs, after one CS has previously been associated with a US (Debiec et al., 2006; Gewirtz and Davis, 1998, 2000; Nader and LeDoux, 1999). It is conceivable that the blockade of the second order fear conditioning could be due to the disruption of LA recurrent connectivity which is mediated in part by NMDA receptors (Johnson and LeDoux, 2004; Johnson et al., 2008).

\section{FUTURE DIRECTIONS}

Hebb's first postulate has been tremendously influential in the neurobiology of learning and memory. In contrast variables in the second postulate 'reverberation' and 'recurrent network' have been harder to define and measure. Nonetheless the idea of memory formation being facilitated by reverberating networks may explain how temporally divergent but behaviorally relevant neural signals become linked. Hebbian-like reverberating networks in the LAd appear to be structured to facilitate coincident associations between temporally divergent cortical and subcortical sensory afferents. In addition, transmission of activity within the network is NMDA receptor dependent and the synapses at each node in the network are plastic. These data raise important questions for future research on how network structure and behavior facilitate the encoding of conditioned fear memory traces (Johnson and LeDoux, 2004; Johnson et al., 2008).

Further studies are needed to specify the degree to which recurrent connectivity converges onto single neurons, and to characterize the precise rules that govern plasticity at network synapses. Many structural and functional questions remain. How does this reverberant activity interact with oscillatory behavior to facilitate sensory and synaptic plasticity? Recent data suggest that the amygdala stores fear memories with much more sensory detail than once supposed (Debiec et al., 2006; Doyère et al., 2007) which raises important questions about how detailed memory traces are encoded by LA networks. For example, does the reverberatory activity participate in the refinement of the LA memory trace? Does nociceptive information transmitted from the thalamus directly to the LA converge in the LA with auditory information from the auditory cortex? Does sensory information from the different senses also converge in the LA and thereby undergo associative plasticity using the thalamic and cortical routes to the LA? While more research is needed on the functional behavior of Hebbian ensembles, recent studies of LA networks have opened a door into the next level of analysis of emotional memory (Johnson et al., 2008).

\section{ACKNOWLEDGMENTS}

This research is supported by: a NSF-CNRS International Cooperative Grant (LRJ, VD, JEL); a CNRS PICS grant (VD, JEL); a CNRS-UPSNYU collaboration grant EmoTime (VD, JEL); a EU-FP6 grant Promemoria (VD); NIH grants MH58911, MH46516, MH38774, MH00956 (JEL); a NARSAD Young Investigator Award and USU grants 208867 and G188K (LRJ). 


\section{REFERENCES}

Armony, J. L., Quirk, G. J., and LeDoux, J. E. (1998). Differential effects of amygdala lesions on early and late plastic components of auditory cortex spike trains during fear conditioning. J. Neurosci. 18, 2592-2601.

Armony, J. L., Servan-Schreiber, D., Cohen, J. C., and LeDoux, J. E. (1996). Emotion and cognition interactions in the thalalmo-cortico-amygdala network: theory and model. Cogn. Neurosci. Soc. Abstr. 3, 76.

Armony, J. L., Servan-Schreiber, D. Cohen, J. D., and LeDoux, J. E. (1995). An anatomically constrained neural network model of fear conditioning. Behav. Neurosci. 109, 246-257.

Armony, J. L., Servan-Schreiber, D., Romanski, L. M., Cohen, J. D., and LeDoux, J. E. (1997). Stimulus generalization of fear responses: effects of auditory cortex lesions in a computational model and in rats. Cereb. Cortex 7, 157-165.

Cruikshank, S. J., Rose, H. J., and Metherate, R. (2002). Auditory thalamocortical synaptic transmission in vitro. J. Neurophysiol. 87, 361-384.

Debiec, J., Doyère, V., Nader, K., and Ledoux, J. E. (2006). Directly reactivated, but not indirectly reactivated, memories undergo reconsolidation in the amygdala. Proc. Natl. Acad. Sci. U.S.A. 103, 3428-3433.

Doron, N. N., and Ledoux, J. E. (1999). Organization of projections to the lateral amygdala from auditory and visual areas of the thalamus in the rat. J. Comp. Neurol. 412, 383-409.

Doron, N. N., and Ledoux, J. E. (2000). Cells in the posterior thalamus project to both amygdala and temporal cortex: a quantitative retrograde double-labeling study in the rat. $J$. Comp. Neurol. 425, 257-274.

Douglas, R. J., Koch, C., Mahowald, M., Martin, K.A., and Suarez, H.H. (1995). Recurrent excitation in neocortical circuits. Science 269, 981-985.

Douglas, R. J., Mahowald, M., Martin, K. A., and Stratford, K. J. (1996). The role of synapses in cortical computation. J. Neurocytol. 25, 893-911.

Doyère, V., Debiec, J., Monfils, M. H., Schafe, G. E., and LeDoux, J. E. (2007). Synapse-specific reconsolidation of distinct fear memories in the lateral amygdala. Nat. Neurosci. 10, 414-416.

Fanselow, M. S., and LeDoux, J. E. (1999). Why we think plasticity underlying Pavlovian fear conditioning occurs in the basolateral amygdala. Neuron 23, 229-232.

Gewirtz, J. C., and Davis, M. (1997). Second-order fear conditioning pre- vented by blocking NMDA receptors in amygdala. Nature 388, 471-474.

Gewirtz, J. C., and Davis, M. (1998). Application of Pavlovian higher-order conditioning to the analysis of the neural substrates of fear conditioning. Neuropharmacology 37, 453-459.

Gewirtz, J.C., and Davis, M. (2000). Using pavlovian higher-order conditioning paradigms to investigate the neural substrates of emotional learning and memory. Learn. Mem. 7, 257-266.

Goense, J. B., and Logothetis, N. K. (2008). Neurophysiology of the BOLD fMRI signal in awake monkeys. Curr. Biol. $18,631-640$

Hebb, D. O. (1949). Organization of Behavior: A Neuropsychological Theory. New York, John Wiley and Sons.

Herry, C., Ciocchi, S., Senn, V., Demmou, L., Muller, C., and Luthi, A. (2008). Switching on and off fear by distinct neuronal circuits. Nature 454, 600-606.

Humeau, Y., Herry, C., Kemp, N., Shaban, H., Fourcaudot, E., Bissiere, S., and Luthi, A. (2005). Dendritic spine heterogeneity determines afferentspecific Hebbian plasticity in the amygdala. Neuron 45, 119-131.

Johnson, L. R.,Hou, M., Ponce-Alvarez, A., Gribelyuk, L. M., Alphs, H. H., Albert, L., Brown, B. L., Ledoux, J. E., and Doyere, V. (2008). A recurrent network in the lateral amygdala: a mechanism for coincidence detection. Front. Neural Circuits 2, 3.

Johnson, L. R., and LeDoux, J. E. (2004). The anatomy of fear: microcircuits of the lateral amygdala. In Fear and Anxiety: The Benefits of Translational Research, J.M. Gorman, ed (Washington D.C., APPA Press), pp. 227-250.

Katzner, S., Nauhaus, I., Benucci, A. Bonin, V., Ringach, D. L., and Carandini, M. (2009). Local origin of field potentials in visual cortex. Neuron 61, 35-41.

Klausberger, T., Marton, L. F., Baude, A., Roberts, J. D., Magill, P. J., and Somogyi, P. (2004). Spike timing of dendrite-targeting bistratified cells during hippocampal network oscillations in vivo. Nat. Neurosci. 7, 41-47.

Kudoh, M., and Shibuki, K. (1997). Importance of polysynaptic inputs and horizontal connectivity in the generation of tetanus-induced longterm potentiation in the rat auditory cortex. J. Neurosci. 17, 9458-9465.

LeDoux, J. (2003). The emotional brain, fear, and the amygdala. Cell. Mol. Neurobiol. 23, 727-738.

LeDoux, J. E. (2000). Emotion circuits in the brain. Annu. Rev. Neurosci. 23 155-184.
LeDoux, J. E., Farb, C., and Romanski, L. (1991). Overlapping projections to the amygdala and striatum from auditory processing areas of the thalamus and cortex. Neurosci. Lett. 134, 139-144.

LeDoux, J. E., Farb, C., and Ruggiero, D.A. (1990). Topographic organization of neurons in the acoustic thalamus that project to the amygdala. J. Neurosci. 10, 1043-1054.

Li,X. F.,Stutzmann, G.E., and LeDoux, J. E. (1996). Convergent but temporally separated inputs to lateral amygdala neurons from the auditory thalamus and auditory cortex use different postsynaptic receptors: in vivo intracellular and extracellular recordings in fear conditioning pathways. Learn. Mem. 3, 229-242.

Maren, S., and Quirk, G. J. (2004) Neuronal signalling of fear memory. Nat. Rev. Neurosci. 5, 844-852.

Mazzoni, A., Panzeri, S., Logothetis, N. K. and Brunel, N. (2008). Encoding of naturalistic stimuli by local field potential spectra in networks of excitatory and inhibitory neurons. PLoS Comput. Biol. 4, e1000239.

Mitzdorf, U. (1985). Current sourcedensity method and application in cat cerebral cortex: investigation of evoked potentials and EEG phenomena. Physiol. Rev. 65, 37-100.

Nader, K., and LeDoux, J. E. (1999) Inhibition of the mesoamygdala dopaminergic pathway impairs the retrieval of conditioned fear associations. Behav. Neurosci. 113 , 891-901.

Nakazawa, K., Quirk, M. C. Chitwood, R. A., Watanabe, M. Yeckel, M. F., Sun, L. D., Kato, A., Carr, C. A., Johnston, D., Wilson, M. A., and Tonegawa, S. (2002). Requirement for hippocampal CA3 NMDA receptors in associative memory recall. Science 297, 211-218.

Pare, D., Collins, D. R., and Pelletier, J. G. (2002). Amygdala oscillations and the consolidation of emotional memories. Trends Cogn. Sci. 6, 306-314.

Pare, D., Quirk, G. J., and Ledoux, J. E. (2004). New vistas on amygdala networks in conditioned fear. J. Neurophysiol. 92, 1-9.

Pelletier, J. G., and Pare, D. (2002). Uniform range of conduction times from the lateral amygdala to distributed perirhinal sites. J. Neurophysiol. 87, 1213-1221.

Pesaran, B. (2009). Uncovering the mysterious origins of local field potentials. Neuron 61, 1-2.

Pesaran, B., Pezaris, J. S., Sahani, M. Mitra, P. P., and Andersen, R. A. (2002). Temporal structure in neuronal activity during working memory in macaque parietal cortex. Nat. Neurosci. 5, 805-811.
Quirk,G.J.,Armony, J.L., and LeDoux, J. E. (1997). Fear conditioning enhances different temporal components of tone-evoked spike trains in auditory cortex and lateral amygdala. Neuron 19, 613-624.

Rao, R. P., and Sejnowski, T. J. (2001). Predictive learning of temporal sequences in recurrent neocortical circuits. Novartis Found. Symp. 239, 208-229; discussion 229-240.

Romanski, L. M., Clugnet, M. C. Bordi, F., and LeDoux, J. E. (1993). Somatosensory and auditory convergence in the lateral nucleus of the amygdala. Behav. Neurosci. 107, 444-450.

Romanski, L. M., and LeDoux, J. E. (1992). Equipotentiality of thalamo-amygdala and thalamo-cortico-amygdala circuits in auditory fear conditioning. J. Neurosci. 12, 4501-4509.

Sah, P., Westbrook, R. F., and Luthi, A. (2008). Fear conditioning and longterm potentiation in the amygdala: what really is the connection? Ann.N.Y. Acad. Sci. 1129, 88-95.

Seidenbecher, T., Laxmi, T. R., Stork, O., and Pape, H.C. (2003). Amygdalar and hippocampal theta rhythm synchronization during fear memory retrieval. Science 301, 846-850.

Sejnowski, T. J. (1999). The book of Hebb. Neuron 24,773-776.

Tegner, J., Compte, A., and Wang, X. J. (2002). The dynamical stability of reverberatory neural circuits. Biol. Cybern. 87, 471-481.

Wang, X. J. (2001). Synaptic reverberation underlying mnemonic persistent activity. Trends Neurosci. 24, 455-463.

Wong, K. F., and Wang, X. J. (2006). A recurrent network mechanism of time integration in perceptual decisions. J. Neurosci. 26, 1314-1328

Conflict of Interest Statement: The authors declare that the research was conducted in the absence of any commercial or financial relationships that could be construed as a potential conflict of interest.

Received:09 May 2009; paperpending published: 26 June 2009; accepted:09 July 2009; published: 15 September 2009.

Citation: Front. Neurosci. (2009) 3, 2: 198 205. doi: 10.3389/neuro.01.027.2009 Copyright (C) 2009 Johnson, LeDoux and Doyère. This is an open-access article subject to an exclusive license agreement between the authors and the Frontiers Research Foundation, which permits unrestricted use, distribution, and reproduction in any medium, provided the original authors and source are credited. 